\title{
Probability of Position and Motion Parameters Estimation for a Radio Beacon in Passive Search and Rescue Systems
}

\author{
Semen Yukhno ${ }^{1}$, Yuriy Petrov ${ }^{2}$, Stanislav Rudyka $^{3}$ \\ ${ }^{1}$ Baltic State Technical University «VOENMEH», St. Petersburg, 190005, Russia \\ ${ }^{2}$ Baltic State Technical University «VOENMEH», St. Petersburg, 190005, Russia, \\ ${ }^{3}$ Baltic State Technical University «VOENMEH», St.Petersburg, 190005, Russia,
}

\begin{abstract}
In this article, the method to estimate the range to the beacon in passive search and rescue systems is proposed. The range to the beacon is determined by applying a set of Kalman filters to the bearing measurements with different hypotheses of the beacon motion and subsequent selection of the hypothesis with the minimum sum of residual errors in target range estimation. The expressions for the expectation and standard deviation of the prediction errors are derived and the probability of selection the correct hypothesis is given. The proposed method allows to evaluate the measurement time, required to determine the range to the beacon with probability $0.9 \ldots 0.95$, which agrees well with statistical analysis of multiple data sets.
\end{abstract}

Keywords: (Multiple) Hypotheses testing, Kalman filter, Probability, Passive radar, Ranging.

\section{INTRODUCTION}

Recent developments in passive or semi-active radars have expanded their application to target location along with active radars. The modern element base has significantly improved the accuracy of determining both the direction of the beacon and improve the accuracy of determining the characteristics of its radiation (carrier frequency, duration and muls and its period). The computing power of the equipment has also increased.

Improving the accuracy of determining the radio characteristics of the signal allows you to perform the identification of the signal at two points of reception with a higher probability, which gave more development for the methods of determining the location of the target by existing methods requires either two passive radars placed at different positions [1; Kevin, et. El Republic., 2010; 23; 3; 26; 9]. Improved performance of processors and integrated circuits made it possible to perform more complex signal processing, which made it possible to perform target detection by an external signal (GSM or broadcast signal) $[2 ; 7 ; 16 ; 9 ; 15 ; 18]$. However, if the target emits, it is possible to determine its range by measuring the target bearing and its radiation parameters using only one mobile passive locator $[10 ; 4 ; 22]$.

The determination of the range must be carried out with some specified accuracy. In these methods, acceptable location accuracy is not achieved after one measurement, but after a while, and is therefore unknown. As data accumulate, the accuracy of the range measurement will increase, and there is a need to determine at what point in time the estimated target location is accurate with a given accuracy.

This article displays the probability of the correct location of the target (emitting beacon) using the algorithm described below $[8 ; 6]$.

\section{MODEL AND METHOD}

To determine the location of a mobile beacon using only one mobile direction finder, the hypothesis testing algorithm has been proposed $[12 ; 20]$. The direction finder moves at speed $\mathrm{V}_{1}$ and course $\Psi_{1}$ when the radiation of the beacon is detected. After the time $\mathrm{T}_{\mathrm{cm}}$ the finder changes the course to $\Psi_{2}$ and continue its movement at speed $\mathrm{V}_{2}[14 ; 13 ; 17]$. Multilevel prognosis of logistics chains in case of uncertainty: Journal of Open Innovation: Technology, Market, and Complexity, 4(1) doi:10.1186/s40852-018-0081-8. The beacon during the measurement time moves rectilinearly with a constant speed. It is required to determine the range to the beacon with the required relative accuracy $\delta \mathrm{D}$. The geometry of the problem is shown in Figure 1. 


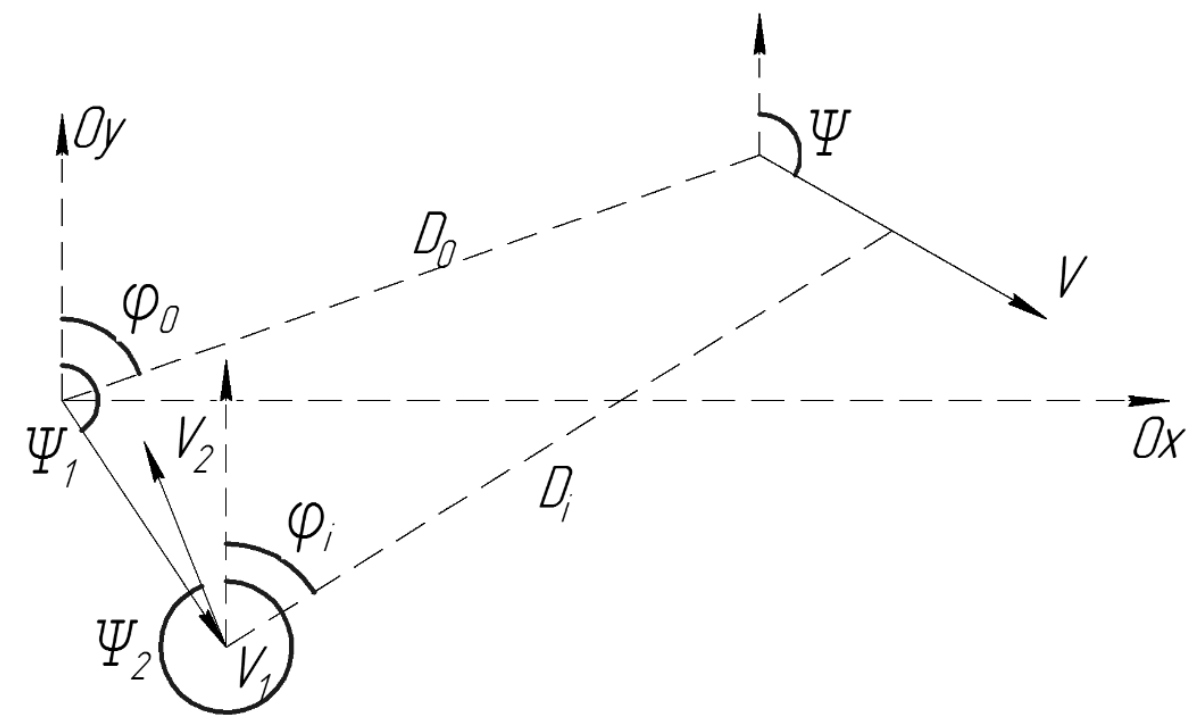

Figure 1: The geometry of the mutual motion of the direction finder and beacon

The notations in Figure 1 are the following: $V_{1}, V_{2}-$ the speed of the finder, $\Psi_{1}, \Psi_{2}$ - the course of the finder movement, V, $\Psi-$ speed and heading of the beacon, $D_{0}, \varphi_{0}-$ range and bearing to the beacon at the moment of its detection, $D_{i}, \varphi_{i}-$ the distance and bearing to the beacon at time i. Oy, Ox - axes of coordinates, the axe Oy directed to the North, the axe Ox directed to the East.

To determine the range to the beacon, a set of onedimensional Kalman filters is formed [24], each filter is determined by a set of hypotheses about the initial range to the beacon, the speed and the course of its movement. Each Kalman filter is denoted by three indices abc $(\mathrm{a}-$ the index of initial range hypotheses, $\mathrm{b}$ - the index of speed hypotheses , c - the index of course hypotheses).

The Kalman filter with indices abc calculates:

1) Prediction of the beacon bearing:

$$
\varphi_{i / i-1}^{a b c}=\hat{\varphi}_{i-1}^{a b c}+\frac{V^{b} \cdot d t \cdot \sin \left(\Psi^{c}-\hat{\varphi}_{i-1}^{a b c}\right)-\sqrt{\left(\Delta x_{i}\right)^{2}+\left(\Delta y_{i}\right)^{2}} \cdot \sin \left(\arg \left(\frac{\Delta x_{i}}{\Delta y_{i}}\right)-\hat{\varphi}_{i-1}^{a b c}\right)}{\hat{D}_{i-i}^{a b c}+V^{b} \cdot d t \cdot \cos \left(\Psi^{c}-\hat{\varphi}_{i-1}^{a b c}\right)-\sqrt{\left(\Delta x_{i}\right)^{2}+\left(\Delta y_{i}\right)^{2}} \cdot \cos \left(\arg \left(\frac{\Delta x_{i}}{\Delta y_{i}}\right)-\hat{\varphi}_{i-1}^{a b c}\right)}
$$

2) Predicted MSE error: $p_{i / i-1}=p_{i-1}$

3) Kalman Gain: $K_{i}=\frac{p_{i / i-1}}{p_{i / i-1}+\sigma_{\varphi}^{2}}$

4) Correction of the beacon bearing: $\hat{\varphi}_{i}^{a b c}=\varphi_{i / i-1}^{a b c}+K_{i} \cdot\left(\widetilde{\varphi}_{i}-\varphi_{i / i-1}^{a b c}\right)$

5) MSE: $p_{i}=\left(1-K_{i}\right) \cdot p_{i / i-1}$

$\mathrm{V}^{\mathrm{b}}$ - the hypothesis on the beacon speed, $\Psi^{\mathrm{c}}$ - the hypothesis on the beacon course, $\hat{D}_{0}^{a b c}=D^{a}$ - the hypothesis on the initial range to the beacon, $\mathrm{V}$ beacon speed, $\Psi$ - the rate of motion of the beacon, $\mathrm{dt}$ - the time between bearing measurements, $\Delta x_{i}-$ movement of the direction finder along the Ox axis during the time dt at step i, $\Delta y_{i}$ - movement of the direction finder along the Oy axis during the time dt at step i.

For each filter, the sum of squared residual errors is calculated

$$
S_{i}=\sum_{k=1}^{i}\left(\tilde{\varphi}_{j}-\hat{\varphi}_{j}\right)^{2}
$$


The estimation of the beacon range and motion parameters is obtained as the hypothesis, for which the corresponding Kalman has the minimal sum of squared residual errors.

\subsection{Probability of correct range hypothesis selection with a given relative accuracy.}

The parameter that determines the choice of the hypothesis with the current range to the target and the parameters of the target movement is the sum of squares of residuals $S_{i}$ at time $i$. Thus, the probability of correct range hypothesis selection at step $i$ of the algorithm is the probability that the sum of the squares of the residuals $S_{i}^{0}$ for the hypothesis with indices abc at the current range to the beacon with the sum of squared

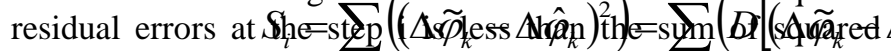
residual errors $S_{i}^{1}$ for tkélhypothesis with indides' a'b'c'. Here the hypothesis with indices a'b'c' has the minimum sum of squared residual errors out of all the hypotheses with the current range estimation outside of the interval $\left[D_{i}^{a b c} \cdot(1-2 \cdot \delta D) ; D_{i}^{a b c} \cdot(1+2 \cdot \delta D)\right]$. The probability of correct range hypothesis selection the becomes:

$p\left(S_{i}^{0}<S_{i}^{1}\right)=\int_{-\infty-\infty}^{+\infty} \int_{0}^{h} f_{0}(x) \cdot d x \cdot f_{1}(h) \cdot d h=\int_{-\infty}^{+\infty} F_{0}(h) \cdot f_{1}(h) \cdot d h$

$f_{0}(x)$ - probability density function (PDF) of the sum of squared residual errors $S_{i}^{0}, f_{1}(h)-$ PDF of the sum of squared residual errors $S_{i}^{1}, F_{0}(h)$ - cumulative density function $(\mathrm{CDF})$ of the sum of squared residual errors $S_{i}^{0}$.

Sum of squared residuals at the i-th step of the algorithm is $S_{i}=\sum_{k=1}^{i}\left(\tilde{\varphi}_{j}-\hat{\varphi}_{j}\right)^{2}=\sum_{k=1}^{i}\left(\varphi_{j}+\Delta \tilde{\varphi}_{j}-\varphi_{j}-\Delta \hat{\varphi}_{j}\right)^{2}=\sum_{k=1}^{i}\left(\Delta \tilde{\varphi}_{j}-\Delta \hat{\varphi}_{j}\right)^{2}$ $\hat{\varphi}_{i}=K_{i} \cdot \tilde{\varphi}_{i}+\left(1-K_{i}\right) \cdot \hat{\varphi}_{i / i-1}=K_{i} \cdot \varphi_{i}+K_{i} \cdot \Delta \tilde{\varphi}_{i}+\left(1-K_{i}\right) \cdot\left(\hat{\varphi}_{i-1}+\hat{u}_{i}\right)=$ $=K_{i} \cdot \varphi_{i}+K_{i} \cdot \Delta \tilde{\varphi}_{i}+\left(1-K_{i}\right) \cdot\left(\varphi_{i-1}+\Delta \hat{\varphi}_{i-1}+\varphi_{i}-\varphi_{i-1}+\Delta u_{i}\right)=$ $=K_{i} \cdot \varphi_{i}+K_{i} \cdot \Delta \tilde{\varphi}_{i}+\left(1-K_{i}\right) \cdot \varphi_{i}+\left(1-K_{i}\right) \cdot \Delta \hat{\varphi}_{i-1}+\left(1-K_{i}\right) \cdot \Delta u_{i}=$ $=\varphi_{i}+K_{i} \cdot \Delta \tilde{\varphi}_{i}+\left(1-K_{i}\right) \cdot \Delta \hat{\varphi}_{i-1}+\left(1-K_{i}\right) \cdot \Delta u_{i}$ $K_{i}$ - Kalman Gain, $\hat{\varphi}_{i / i-1}=\hat{\varphi}_{i-1}+\hat{u}_{i}$ - prediction of bearing to the beacon, $\hat{u}_{i}=\varphi_{i}-\varphi_{i-1}+\Delta u_{i}-$ control action, $\varphi_{i}-$ the true value of bearing, $\Delta u_{i}-$ prediction error.

Bearing estimation error is equal to:

$\Delta \hat{\varphi}_{i}=\hat{\varphi}_{i}-\varphi_{i}=\varphi_{i}+K_{i} \cdot \Delta \tilde{\varphi}_{i}+\left(1-K_{i}\right) \cdot \Delta \hat{\varphi}_{i-1}+\left(1-K_{i}\right) \cdot \Delta u_{i}-\varphi_{i}=$ $=K_{i} \cdot \Delta \tilde{\varphi}_{i}+\left(1-K_{i}\right) \cdot \Delta \hat{\varphi}_{i-1}+\left(1-K_{i}\right) \cdot \Delta u_{i}=K_{i} \cdot \Delta \tilde{\varphi}_{i}+\left(1-K_{i}\right) \cdot K_{i-1} \cdot \Delta \tilde{\varphi}_{i-1}+$

$+\ldots+\left(1-K_{i}\right) \cdot \ldots \cdot\left(1-K_{2}\right) \cdot K_{1} \cdot \Delta \tilde{\varphi}_{1}+\left(1-K_{i}\right) \cdot \ldots \cdot\left(1-K_{1}\right) \cdot \Delta \tilde{\varphi}_{0}+$ $+\left(1-K_{i}\right) \cdot \Delta u_{i}+\left(1-K_{i}\right) \cdot\left(1-K_{i-1}\right) \cdot \Delta u_{i-1}+\ldots+\left(1-K_{i}\right) \cdot \ldots \cdot\left(1-K_{1}\right) \cdot \Delta u_{1}$

If the variance of the noise after filtering is zero $(\mathrm{Q})$, than Kalman Gain is equal to $K_{i}=(i+1)^{-1}$, and the expression for the bearing estimation error takes the following form:

$$
\Delta \hat{\varphi}_{i}=\sum_{k=0}^{i} \frac{\Delta \tilde{\varphi}_{k}}{i+1}+\sum_{k=1}^{i} \frac{k}{i+1} \cdot \Delta u_{k}=\sum_{k=0}^{i} \frac{\Delta \tilde{\varphi}_{k}}{i+1}+U_{i}
$$

, $\tilde{\varphi}_{j}-$ where $\varphi_{j}$ - the bearing of the radio beacon, $\tilde{\varphi}_{j}-$ the measured bearing of the beacon, $\hat{\varphi}_{j}$ - the estimated bearing of the beacon , $\Delta \tilde{\varphi}_{j}$ - the measurement error in the bearing of the radio beacon, $\Delta \hat{\varphi}_{j}$ - estimation error of the bearing to the beacon.

The sum of the squares of residuals for the $\mathrm{i}$-th reference is a random value in the form

$$
S_{i}=\sum_{k=1}^{i}\left(\left(\Delta \tilde{\varphi}_{k}-\Delta \hat{\varphi}_{k}\right)^{2}\right)=\sum_{k=1}^{i}\left(D\left[\left(\Delta \tilde{\varphi}_{k}-\Delta \hat{\varphi}_{k}\right)^{2}\right] \cdot(N(0,1))^{2}\right)=\sum_{k=1}^{i}
$$

$N(0,1)$ - random variable with standard normal distribution, $\chi^{2}(1)$ a random variable distributed by the Chi-square law with one degree of freedom.

To solve the problem of determining the location of the radio beacon, it is necessary to collect more than 100 measurements of the direction finder. Then, according to the Central limit theorem, the sum of the squares of residuals can be considered a normally distributed random variable $[25 ; 21]$.

The probability density of the normal distribution is determined by the parameters of expectation and variance. We derive the expectation and variance of the sum of squares of residuals with the minimum sum of squares of residuals $S_{i}^{0}$ (no prediction errors) and prediction errors $S_{i}^{1}$.

Kalman filter allows to use the following expression for the estimate of the bearing at the time $i$ : 
Control action $\mathrm{U}_{\mathrm{i}}$ on step $\mathrm{i}$ is equal to:

$u_{i}=\left\{V \cdot d t \cdot \sin \left(\Psi-\varphi_{i-1}\right)-\sqrt{\left(\Delta x_{i}\right)^{2}+\left(\Delta y_{i}\right)^{2}} \cdot \sin \left[\arg \left(\Delta x_{i} / \Delta y_{i}\right)-\varphi_{i-1}\right]\right\} / D_{i}=A_{i} / D_{i}$, where

$D_{i}=D_{i-1}+V \cdot d t \cdot \cos \left(\Psi-\varphi_{i-1}\right)-\sqrt{\left(\Delta x_{i}\right)^{2}+\left(\Delta y_{i}\right)^{2}} \cdot \cos \left(\arg \left(\Delta x_{i} / \Delta y_{i}\right)-\varphi_{i-1}\right)-$ the value of the current range to the beacon, $\mathrm{D}_{0}-$ the initial range to the target.

The estimated control action (in case of errors) for the hypothesis with indices abc, provided $\cos \hat{\varphi}_{i} \approx \cos \varphi_{i}$ и $\sin \hat{\varphi}_{i} \approx \sin \varphi_{i}$, is equal:

$\hat{u}_{i}^{a b c}=\left\{V^{b} \cdot d t \cdot \sin \left(\Psi^{c}-\hat{\varphi}_{i-1}^{a b c}\right)-\sqrt{\left(\Delta x_{i}\right)^{2}+\left(\Delta y_{i}\right)^{2}} \cdot \sin \left[\arg \left(\Delta x_{i} / \Delta y_{i}\right)-\hat{\varphi}_{i-1}^{a b c}\right]\right\} / \hat{D}_{i}^{a b c}=$ $=\left\{V^{b} \cdot d t \cdot \sin \left(\Psi^{c}-\varphi_{i-1}\right)-\sqrt{\left(\Delta x_{i}\right)^{2}+\left(\Delta y_{i}\right)^{2}} \cdot \sin \left[\arg \left(\Delta x_{i} / \Delta y_{i}\right)-\varphi_{i-1}\right]\right\} / \hat{D}_{i}^{a b c}=\hat{A}_{i}^{a b c} / \hat{D}_{i}^{a b c}$

The error of the control action is equal to:

$\Delta u_{i}=\hat{A}_{i}^{a b c} / \hat{D}_{i}^{a b c}-A_{i} / D_{i}$

In what follows, notation $\Delta \hat{\varphi}_{i}$ will be used for the error of bearing estimation in case of accurate prediction, i.e. $\mathrm{U}_{\mathrm{i}}=0$.

The bearing measurement error has a normal distribution with zero mean and variance $\sigma_{\varphi}^{2}\left(M\left[\Delta \tilde{\varphi}_{i}\right]=0, D\left[\Delta \tilde{\varphi}_{i}\right]=\sigma_{\varphi}^{2}\right)$. The bearing measurement are considered to be uncorrelated.

\subsection{The sum of squares of residuals without error prediction}

The expectation and the variance of the bearing estimation error for accurate prediction are:

$M\left[\Delta \hat{\varphi}_{i}\right]=M\left\lfloor\frac{1}{i+1} \cdot \Delta \tilde{\varphi}_{i}+\ldots+\frac{1}{i+1} \cdot \Delta \tilde{\varphi}_{0}\right\rfloor=M\left\lfloor\frac{1}{i+1} \cdot \Delta \tilde{\varphi}_{i}\right\rfloor+\ldots+M\left\lfloor\frac{1}{i+1} \cdot \Delta \tilde{\varphi}_{0}\right\rfloor=0$

$D\left[\Delta \hat{\varphi}_{i}\right]=M\left[\left(\frac{1}{i+1} \cdot \Delta \tilde{\varphi}_{i}+\ldots+\frac{1}{i+1} \cdot \Delta \tilde{\varphi}_{0}\right)^{2}\right\rfloor-M^{2}\left[\Delta \hat{\varphi}_{i}\right]=\frac{\sigma_{\varphi}^{2}}{i+1}$

The sum of squared residual errors for accurate forecasting $(\mathrm{Uj}=0)$ is equal:

$S_{i}^{0}=\sum_{j=1}^{i}\left(\Delta \tilde{\varphi}_{j}-\Delta \hat{\varphi}_{j}\right)^{2}$

We define the mean of sum of squared residual errors:

$M\left[S_{i}^{0}\right]=M\left[\sum_{j=1}^{i}\left(\Delta \tilde{\varphi}_{j}-\Delta \hat{\varphi}_{j}\right)^{2}\right]=M\left[\left(\Delta \tilde{\varphi}_{i}-\Delta \hat{\varphi}_{i}\right)^{2}\right]+\ldots+M\left[\left(\Delta \tilde{\varphi}_{1}-\Delta \hat{\varphi}_{1}\right)^{2}\right]=\sigma_{\varphi}^{2} \cdot\left(\sum_{j=1}^{i} \frac{j}{j+1}\right)$ 
Determine the variance of the sum of squared residual errors:

$$
\begin{aligned}
& D\left[S_{i}^{0}\right]=M\left[\left(\sum_{k=1}^{i}\left(\Delta \widetilde{\varphi}_{k}-\Delta \hat{\varphi}_{k}\right)^{2}\right)^{2}\right\rfloor-\left(M\left[S_{i}^{0}\right]\right)^{2}=M\left[\sum_{k=1}^{i}\left(\Delta \widetilde{\varphi}_{k}-\Delta \hat{\varphi}_{k}\right)^{4}+\right. \\
& \left.+2 \cdot \sum_{k=2}^{i} \sum_{j=1}^{k-1}\left(\Delta \widetilde{\varphi}_{k}-\Delta \hat{\varphi}_{k}\right)^{2} \cdot\left(\Delta \widetilde{\varphi}_{j}-\Delta \hat{\varphi}_{j}\right)^{2}\right]-\left(M\left[S_{i}^{0}\right]\right)^{2}= \\
& =\sigma_{\varphi}^{4} \cdot\left(\sum_{k=1}^{i} \frac{4 \cdot k^{2}-2 \cdot k}{(k+1)^{3}}-4 \cdot \sum_{k=2}^{i} \sum_{j=1}^{k-1} \frac{j}{(k+1)^{2} \cdot(j+1)}\right)
\end{aligned}
$$

To simplify the calculation of the variance, we approximate it as follows:

$$
D\left[S_{i}^{0}\right]=\sigma_{\varphi}^{4} \cdot \log _{1.645}^{0.294}(i)
$$

\subsection{Sum of squares of residuals with prediction errors}

The sum of squares of residuals with prediction error:

$$
S_{i}^{1}=\sum_{j=1}^{i}\left(\tilde{\varphi}_{j}-\hat{\varphi}_{j}-U_{j}\right)^{2}=\sum_{j=1}^{i}\left(\varphi_{j}+\Delta \tilde{\varphi}_{j}-\varphi_{j}-\Delta \hat{\varphi}_{j}-U_{j}\right)^{2}=\sum_{j=1}^{i}\left(\Delta \tilde{\varphi}_{j}-\Delta \hat{\varphi}_{j}-U_{j}\right)^{2}
$$

Define the expectation of the sum of squared residual errorswith prediction error by:

$$
M\left[S_{i}^{1}\right]=M\left[\sum_{j=1}^{i}\left(\Delta \tilde{\varphi}_{j}-\Delta \hat{\varphi}_{j}-U_{j}\right)^{2}\right]=\sigma_{\varphi}^{2} \cdot \sum_{j=1}^{i} \frac{j}{j+1}+\sum_{j=1}^{i} M\left[U_{j}^{2}\right]
$$

Since the prediction error does not include measurement errors, the expectation of the prediction error is equal to:

$$
M\left[U_{j}^{2}\right]=U_{j}^{2}
$$

Therefore: $M\left[S_{i}^{1}\right]=\sigma_{\varphi}^{2} \cdot \sum_{j=1}^{i} \frac{j}{j+1}+\sum_{j=1}^{i} U_{j}^{2}$.

The variance of the sum of squared residual errors in presence of prediction error is given by:

$$
\begin{aligned}
& D\left[S_{i}^{1}\right]=M\left[\left(\sum_{k=1}^{i}\left(\Delta \tilde{\varphi}_{k}-\Delta \hat{\varphi}_{k}-U_{k}\right)^{2}\right)^{2}\right\rfloor-\left(M\left[S_{i}^{1}\right]\right)^{2}=M\left[\sum_{k=1}^{i}\left(\Delta \tilde{\varphi}_{k}-\Delta \hat{\varphi}_{k}-U_{k}\right)^{4}+\right. \\
& \left.+2 \cdot \sum_{k=2}^{i} \sum_{j=1}^{k-1}\left(\tilde{\varphi}_{k}-\Delta \hat{\varphi}_{k}-U_{k}\right)^{2} \cdot\left(\tilde{\varphi}_{j}-\Delta \hat{\varphi}_{j}-U_{j}\right)^{2}\right]-\left(M\left[S_{i}^{1}\right]\right)^{2}=\sigma_{\varphi}^{4} \cdot\left(\log _{1.645}^{0.147}(i)\right)^{2}+ \\
& +2 \cdot \sigma_{\varphi}^{2} \cdot\left(3 \cdot \sum_{k=1}^{i} \frac{U_{k}^{2} \cdot k}{k+1}+\sum_{k=2}^{i} \sum_{j=1}^{k-1}\left(\frac{k \cdot U_{j}^{2}}{k+1}+\frac{j \cdot U_{k}^{2}}{j+1}-\frac{4 \cdot U_{k} \cdot U_{j}}{(k+1) \cdot(j+1)}\right)-\sum_{k=1}^{i} \frac{k}{k+1} \cdot \sum_{k=1}^{i} U_{k}^{2}\right)
\end{aligned}
$$

\subsection{Probability of correct hypothesis selection}

The hypothesis with the minimum sum of squared residual errors for the case of no prediction error has mathematical expectation and standard deviation equal to:

$$
\begin{aligned}
& M\left[S_{i}^{0}\right]=\sigma_{\varphi}^{2} \cdot \sum_{j=2}^{i+1} \frac{j}{j+1} \\
& \sigma_{S_{i}^{0}}=\sigma_{\varphi}^{2} \cdot \log _{1.645}^{0.147}(i)
\end{aligned}
$$


Mathematical expectation and RMS of the hypothesis with the prediction error are respectively equal to:

$$
\begin{aligned}
& M\left[S_{i}^{1}\right]=\sigma_{\varphi}^{2} \cdot \sum_{j=1}^{i} \frac{j}{j+1}+\sum_{j=1}^{i} U_{j}^{2} \\
& \sigma_{S_{i}^{1}}=\left(\sigma_{\varphi}^{4} \cdot\left(\log _{1.645}^{0.147}(i)\right)^{2}+\right. \\
& \left.+2 \cdot \sigma_{\varphi}^{2} \cdot\left(3 \cdot \sum_{k=1}^{i} \frac{U_{k}^{2} \cdot k}{k+1}+\sum_{k=2}^{i} \sum_{j=1}^{k-1}\left(\frac{k \cdot U_{j}^{2}}{k+1}+\frac{j \cdot U_{k}^{2}}{j+1}-\frac{4 \cdot U_{k} \cdot U_{j}}{(k+1) \cdot(j+1)}\right)-\sum_{k=1}^{i} \frac{k}{k+1} \cdot \sum_{k=1}^{i} U_{k}^{2}\right)\right)^{0.5}
\end{aligned}
$$

To obtain the sum of squares of residuals without prediction errors to the standard normal value, we subtract the value from both sums $M\left[S_{i}^{0}\right]$ and then divide by $\sigma_{S_{i}^{0}}$. As a result, we get:

$$
\begin{aligned}
& M\left[S_{i}^{\prime 0}\right]=0 \\
& \sigma_{S_{i}^{\prime 0}}=1 \\
& M\left[S_{i}^{\prime 1}\right]=\frac{1}{\log _{1.645}^{0.147}(i)} \cdot \sum_{j=1}^{i} U_{j}^{2}
\end{aligned}
$$$$
\sigma_{S^{\prime}{ }_{i}}=\sqrt{1+\frac{2 \cdot \sigma_{\varphi}^{2} \cdot\left(3 \cdot \sum_{k=1}^{i} \frac{U_{k}^{2} \cdot k}{k+1}+\sum_{k=2}^{i} \sum_{j=1}^{k-1}\left(\frac{k \cdot U_{j}^{2}}{k+1}+\frac{j \cdot U_{k}^{2}}{j+1}-\frac{4 \cdot U_{k} \cdot U_{j}}{(k+1) \cdot(j+1)}\right)-\sum_{k=1}^{i} \frac{k}{k+1} \cdot \sum_{k=1}^{i} U_{k}^{2}\right)}{\left(\log _{1.645}^{0.147}(i)\right)^{2}}}
$$

Thus, the expression for calculating the probability of choosing a hypothesis without prediction errors can be written as follows:

$$
p\left(S_{i}^{0}<S_{i}^{1}\right)=\frac{1}{2} \cdot \int_{-\infty}^{+\infty}\left(1+\operatorname{erf}\left(\frac{h}{\sqrt{2}}\right)\right) \cdot \frac{\exp \left(-\frac{\left(h-M\left[S_{i}^{\prime 1}\right]\right)^{2}}{2 \cdot \sigma_{S_{i}^{1}}^{\prime 2}}\right)}{\sigma_{S_{i}^{\prime 1}} \cdot \sqrt{2 \cdot \pi}} \cdot d h
$$

\subsection{Simulations}

To check the efficiency of the proposed method of calculating the probability of correctly determining the range calculated by the formula (1), 20 realizations for each set of parameters were simulated. For each realization, the following parameters of the targets location and movement were set: the speed of movement of the direction finder is $14 \mathrm{~m} / \mathrm{s}$, the initial course of the direction finder is $0^{\circ}$, the changed course $-180^{\circ}$, the time of course change is 600 seconds, the initial bearing of the beacon $90^{\circ}$, the initial ranges to the beacon are 150, 200 and $250 \mathrm{~km}$, the speed of the beacon movement is $10 \mathrm{~m} / \mathrm{s}$, its course is $0^{\circ}$, the bearings are $0.2^{\circ}$ and $0.3^{\circ}$. The number of hypotheses about the initial range is 40 , the minimum range is $40 \mathrm{~km}$, the step of hypotheses is $10 \mathrm{~km}$, the number of hypotheses for the beacon speed is 21 , the initial speed is 0 $\mathrm{m} / \mathrm{s}$, the step of hypotheses is $1 \mathrm{~m} / \mathrm{s}$, the number of hypotheses on the course of the beacon is 72 , the initial value of the course is $0^{\circ}$, the step between hypotheses is $5^{\circ}$.

Simulation results are shown (see figures 2-4), where the graph "frequency" shows what part of the estimations at a time $t$ does not exceed the limit of $\delta_{D}=10 \%$ of the true range.
The figures demonstrate that before the direction finder has changed the course, the probability to determine the range with a given accuracy is low while after the course has changed, the probability to determine the range with a given accuracy rises and approaches one.

The probability graph is calculated by the formula (1) from one realization of a data set. Since there is no a priori information about the range to the target at each step of the algorithm, the true value of the range is taken as the current range estimation of the Kalman filter with the smallest sum of squared residual errors. In this case, the probability of the correct hypothesis selection at any step of the algorithm is at least 0.5 .

Despite the fact that the graphs are different for amsll number of measurments (before the time of the change of the direction finder's course), they have similar behavior for high propbailities of correct range estimation ( $p>0.9)$. 


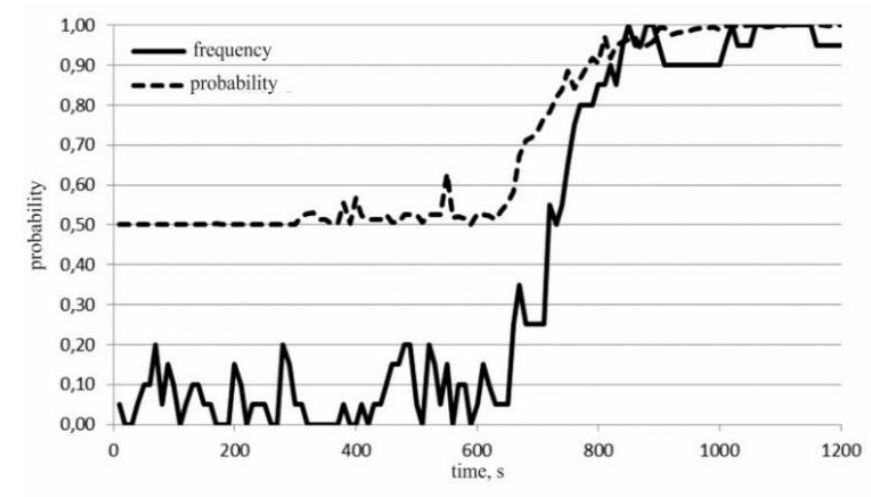

Figure 2: Probability of correct hypothesis selection, the range to the beacon $150 \mathrm{~km}$, the standard deviation of bearing is 0.2

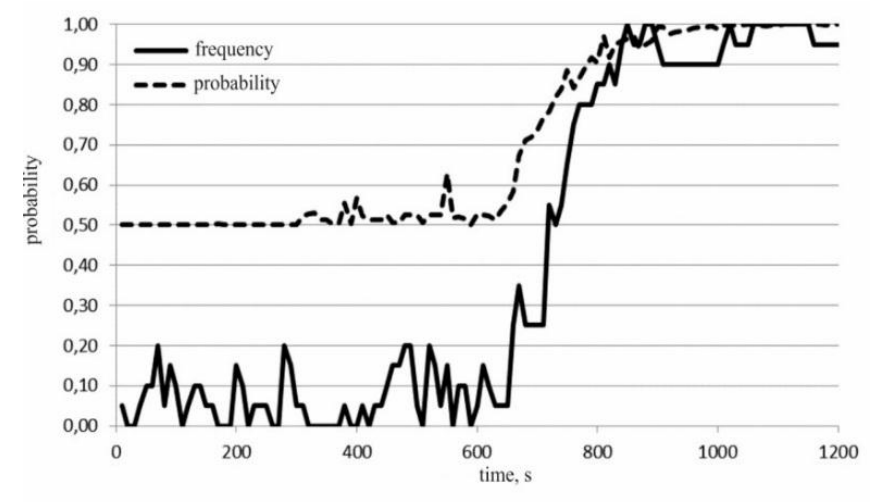

Figure 3: Probability of correct hypothesis selection, the range to the beacon $200 \mathrm{~km}$, the standard deviation of bearing is 0.2 )

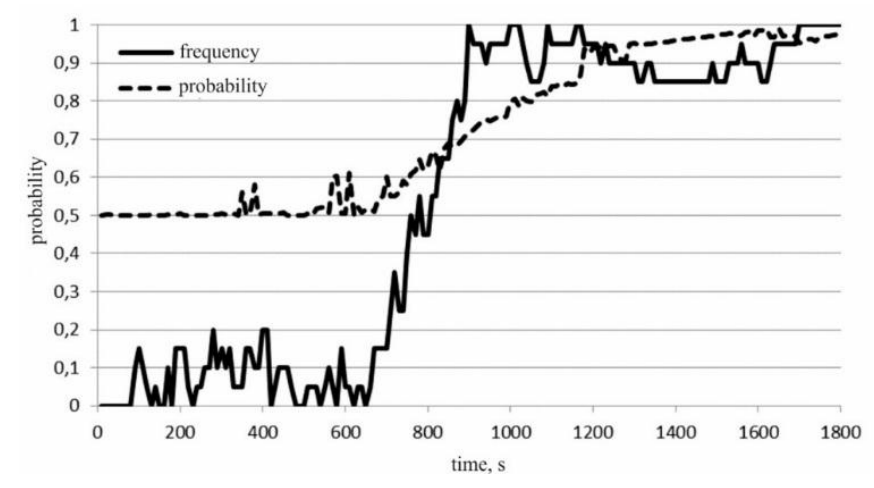

Figure 4: Probability of correct hypothesis selection, the distance to the beacon $250 \mathrm{~km}$, the standard deviation of bearing 0.3

\section{CONCLUSIONS}

Simulation results demonstrate that the provided analytical expression for the probability of determining the range to the beacon with the given accuracy reaches a probability of
0.9..0.95 agrees well with numerical simulations. Therefore, the proposed technique can be considered for determining the location of the beacon with a given probability, in contrast to the methods with a fixed time required to perform the maneuver.

\section{CONFLICT OF INTEREST}

The authors confirm that there is no conflict of interest to declare for this publication

\section{ACKNOWLEDGEMENT}

The work was carried out in accordance with the decree of the Government of the Russian Federation from 09.04.2010 № 218 (PROJECT 218) in the framework of R\&D, executing with the financial support of the Ministry of education and science of the Russian Federation (agreement № 074-11-2018025 from 13.07.2018). Work is performed in lead R\&D performer organization: Federal State Budgetary Educational Institution of Higher Professional Education BSTU "VOENMEH" named after D.F. Ustinov.

\section{REFERENCES}

[1] Bishop, A. N., Fidan, B., Anderson, B. D., Doğançay, K., \& Pathirana, P. N. (2010). Optimality analysis of sensor-target localization geometries. Automatica, 46(3), 479-492.

[2] Coleman, C. J., Watson, R. A., \& Yardley, H. (2008, May). A practical bistatic passive radar system for use with DAB and DRM illuminators. In 2008 IEEE Radar Conference (pp. 1-6). IEEE.

[3] Daun, M., Nickel, U., \& Koch, W. (2012). Tracking in multistatic passive radar systems using DAB/DVB-T illumination. Signal Processing, 92(6), 1365-1386.

[4] Dini, D. H., Jahanchahi, C., \& Mandic, D. P. (2012). Kalman filtering for widely linear complex and quaternion valued bearings only tracking. IET signal processing, 6(5), 435-445.

[5] Olivadese, D., Giusti, E., Petri, D., Martorella, M., Capria, A., \& Berizzi, F. (2013). Passive isar with dvb-t signals. IEEE Transactions on Geoscience and Remote Sensing, 51(8), 4508-4517.

[6] Efanov, D., Sapozhnikov, V., Sapozhnikov, V., \& Plotnikov, D. (2018, September). The Evaluation of Error Detection Probability at the Outputs of Combinational Circuits Under Concurrent Error Detection on the Basis of Summation Codes. In 2018 IEEE East-West Design \& Test Symposium (EWDTS) (pp. 1-5). IEEE.

[7] Falcone, P., Colone, F., Macera, A., \& Lombardo, P. (2012, May). Localization and tracking of moving targets with WiFi-based passive radar. In 2012 IEEE Radar Conference (pp. 0705-0709). IEEE. 
[8] Glazunov, V. V., Kurochkin, M. A., \& Popov, S. G. (2015). Qualification routes messaging for dynamic systems using logical-probabilistic. Nonlinear Phenomena in Complex Systems, 18(1), 99-104.

[9] Gogineni, S., Rangaswamy, M., Rigling, B. D., \& Nehorai, A. (2013). Cramér-Rao bounds for UMTSbased passive multistatic radar. IEEE Transactions on Signal Processing, 62(1), 95-106.

[10] Herman, S., \& Moulin, P. (2002, March). A particle filtering approach to FM-band passive radar tracking and automatic target recognition. In Proceedings, IEEE Aerospace Conference (Vol. 4, pp. 4-4). IEEE.

[11] Chetty, K., Woodbridge, K., Guo, H., \& Smith, G. E. (2010, May). Passive bistatic WiMAX radar for marine surveillance. In 2010 IEEE Radar Conference (pp. 188193). IEEE.

[12] Khachatryan, V., Sirunyan, A. M., Tumasyan, A., Adam, W., Bergauer, T., Dragicevic, M., ... \& Hartl, C. (2015). Observation of the rare B0s $\rightarrow \mu+\mu$-decay from the combined analysis of CMS and LHCb data.

[13] Khasanov, M. M., Lezhnev, K. E., Pashkin, V. D., \& Roshchektaev, A. P. (2018). Application of the new multi-component suspension model for skin-factor evaluating on the wells equipped with gravel packs. Neftyanoe Khozyaystvo - Oil Industry, 2018(12), 6367.

[14] Khludova, M. (2017, May). Evaluation model for the stochastic flowshop cyclic scheduling problem. In 2017 International Conference on Industrial Engineering, Applications and Manufacturing (ICIEAM) (pp. 1-4). IEEE.

[15] Langellotti, D., Colone, F., Lombardo, P., Tilli, E., Sedehi, M., \& Farina, A. (2014, October). Over the horizon maritime surveillance capability of DVB-T based passive radar. In 2014 44th European Microwave Conference (pp. 1812-1815). IEEE.

[16] Li, H. W., \& Wang, J. (2012). Particle filter for manoeuvring target tracking via passive radar measurements with glint noise. IET Radar, Sonar \& Navigation, 6(3), 180-189.

[17] Lukashevich, N., Svirina, A., \& Garanin, D. (2018). Multilevel prognosis of logistics chains in case of uncertainty: information and statistical technologies implementation. Journal of Open Innovation: Technology, Market, and Complexity, 4(1), 2.

[18] Ma, H., Antoniou, M., Pastina, D., Santi, F., Pieralice, F., Bucciarelli, M., \& Cherniakov, M. (2017). Maritime moving target indication using passive GNSS-based bistatic radar. IEEE Transactions on Aerospace and Electronic Systems, 54(1), 115-130.

[19] Mishakova, A. V., Vakhrushkina, A. V., Anishchenko, D. R., \& Tatarkina, Y. A. (2017). Program Evaluation and Review Technique as the tool for time control. Magazine of Civil Engineering, 72(4).
[20] Muravyeva, L., \& Vatin, N. (2016). Elaboration of the method for safety assessment of subsea pipeline with longitudinal buckling. Advances in Civil Engineering, 2016.

[21] Petinov, S. V. (2018). Elements of Statistics and Probability. In In-Service Fatigue Reliability of Structures (pp. 9-20). Springer, Cham.

[22] Setlur, P., Smith, G. E., Ahmad, F., \& Amin, M. G. (2012). Target localization with a single sensor via multipath exploitation. IEEE Transactions on Aerospace and Electronic Systems, 48(3), 1996-2014.

[23] Viani, F., Rocca, P., Benedetti, M., Oliveri, G., \& Massa, A. (2010). Electromagnetic passive localization and tracking of moving targets in a WSNinfrastructured environment. Inverse Problems, 26(7), 074003.

[24] Bishop, G., \& Welch, G. (2001). An introduction to the kalman filter. Proc of SIGGRAPH, Course, 8(2759923175), 41.

[25] Zegzhda, P., Zegzhda, D., Pavlenko, E., \& Dremov, A. (2017, October). Detecting Android application malicious behaviors based on the analysis of control flows and data flows. In Proceedings of the 10th International Conference on Security of Information and Networks (pp. 280-283). ACM.

[26] Zhang, Y. D., \& Himed, B. (2013, April). Moving target parameter estimation and SFN ghost rejection in multistatic passive radar. In 2013 IEEE Radar Conference (RadarCon13) (pp. 1-5). IEEE. 